Case Reports

\title{
Early Management of Non-Syndromic Hypodontia in a Child: Three-Year Follow-Up
}

\author{
Roula Berbari and Nahla Nassif \\ Department of Pediatric Dentistry and Public Health, Faculty of Dental Medicine, Lebanese University, Beirut, Lebanon
}

\author{
Article history \\ Received: 13-02-2021 \\ Revised: 31-03-2021 \\ Accepted: 01-04-2021 \\ Corresponding Author: \\ Roula Berbari \\ Department of Pediatric \\ Dentistry and Public Health, \\ Faculty of Dental Medicine, \\ Lebanese University, Beirut, \\ Lebanon \\ Email: rouberdag@hotmail.com
}

\begin{abstract}
Hypodontia is the congenital absence of few teeth. The early multidisciplinary management of hypodontia assures the reestablishment of the disturbed psychological, aesthetic and oral functions. In this study, the clinical case of a child presenting a non-syndromic hypodontia with three agenesis teeth was described. No evidence of maxillary and mandibular third molars bud formation was noted on the orthopantomograph. To replace the two missing permanent mandibular central incisors, two mini-implants in the mandibular symphyseal region were placed and then restored by individually fixed prostheses. In the maxilla, the permanent canine in a complete transposition, was reshaped with composite resin restoration to replace the missing right permanent lateral incisor. Clinical and radiological reassessments were done 3 years later. The aesthetic and functional evaluations were satisfactory. The marginal bone level was stable with no bone loss. The periodontal health around the miniimplants were confirmed in conjunction with mechanical function.
\end{abstract}

Keywords: Tooth Agenesis, Hypodontia, Mini-Implant

\section{Introduction}

Hypodontia or dental agenesis is the congenital absence of few teeth (Nunn et al., 2003). This dentofacial anomaly exists in isolated or syndromic form with a sporadic or familial mode of transmission (Fauzi et al., 2018). The estimated prevalence of permanent dentition hypodontia ranges from 1.6 to $6.9 \%$. The most frequently affected teeth are the second premolars and the maxillary lateral incisors (Al-Aniet al., 2017).

Agenesis in children can have harmful effects on alveolar growth, phonation and mastication. Since health problem can result, it is important to detect this anomaly as soon as possible, in order to initiate the most appropriate early treatment (Nunn et al., 2003).

This paper aims to report the clinical case of a young patient with dental agenesis and to emphasize the role of the pediatric dentist in the prompt detection of cases of hypodontia. An early multidisciplinary care adapted to his age is established in order to restore the various disturbed functions. Follow-up and reassessment of the case were carried out 3 years later.

\section{Clinical Case}

A healthy nine-year-old boy presented to the Pediatric Dentistry Department at the Faculty of
Dentistry of the Lebanese University. He was complaining of aesthetic and functional problems with discomfort when chewing in the anterior mandibular region. The anamnesis confirmed the absence of trauma, dental avulsion or a family history of agenesis.

Clinical examination, periapical and panoramic radiographs revealed at the maxilla, the complete transposition of the permanent right canine (\#13) to the site of the missing right lateral incisor (\#12) with the presence of the right deciduous canine (\#53). In the mandible, the absence of permanent central incisors (\#31 and \#41) and the persistence of temporary central incisors (\#71 and \#81) in the arch were noted (Fig. 1). No evidence of maxillary and mandibular third molars bud formation was perceived on the orthopantomograph.

Two years later, the patient decided to undergo treatment. Informed consent form was signed by the patient's mother for the dental treatment and for the case report publication.

After discussing with the parents and the patient the treatment options, the treatment plan was elaborated as follows:

- In mandible, extraction of \#71 and \#81, placement of two mini-implants in the mandibular symphyseal region, sealing two single porcelainfused to metal restorations 
- In maxilla: Reshaping the permanent canine (\#13) into lateral incisor

\section{Treatment}

A Cone Beam Computed Tomography (CBCT) was realized to assess bone quality.

The extractions of \#71 and \#81 were carried out.

Six months later, a minimal invasive surgical intervention with a flapless technique and mini-incision was done after lingual infiltration and mental nerve block anesthesia (Septanest, 4\% articaine hydrochloride and adrenaline 1:100 000/Septodont-France). Two MiniImplants (MDI Max, $2.4 \times 13 \mathrm{~mm}, 3 \mathrm{M}$ ESPE USA) with one-piece titanium screw and a square prosthetic head were sited to replace the missing mandibular permanent central incisors.

Since the patient was very cooperative, no sedation was involved.

Recommendations were given to the patient to reduce the oral bacterial load $(0.12 \%$ Chlorhexidinemouthwash/twice a day for one week). Antibiotic was prescribed for five days (Augmentin $625 \mathrm{mg}$ tablet, twice daily).

One month later and after final impression,both mini implants were restored with single porcelain-fused to metal restorations then sealed with resin modified glass ionomer cement (Fig. 2).

At the maxilla, since the patient wanted a conservative treatment and was refusing any orthodontic and prosthetic treatment, a coronoplasty of the permanent canine (\#13) with composite resin restoration $\left(3 \mathrm{M}^{\mathrm{TM}}\right.$ Filtek $^{\mathrm{TM}} \mathrm{Z} 250$ USA) was executed, reshaping it into a lateral incisor (Fig. 3).

The patient was seen 3 years after treatment, at age 15. The orthopantomogram revealed a stable marginal bone level with no bone loss (Fig. 4). The periodontal health around the mini-implants were confirmed in conjunction with mechanical function.

The orthodontic treatment was continued to be refused for financial reasons.
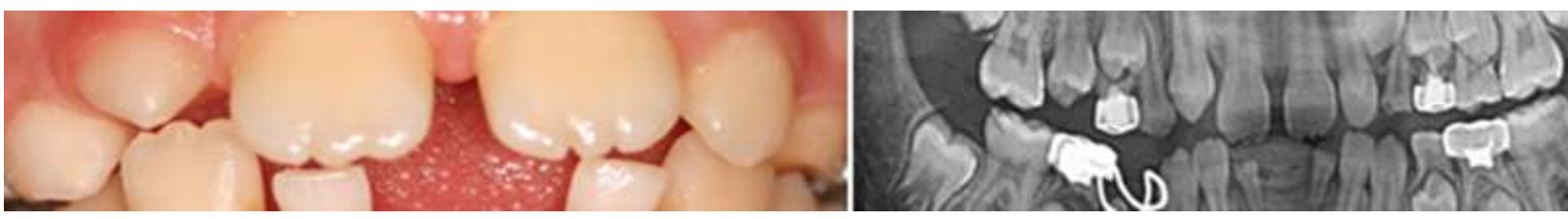

Fig. 1: Intraoral photo at age 9, showing the non-exfoliation of the temporary mandibular central incisors (\#71 and \#81) and the complete transposition of the right permanent maxillary canine (\#13) to the site of the absent permanent lateral incisor (a). Panoramic X-ray showing agenesis of \#12, \#31 and \#41 (b).

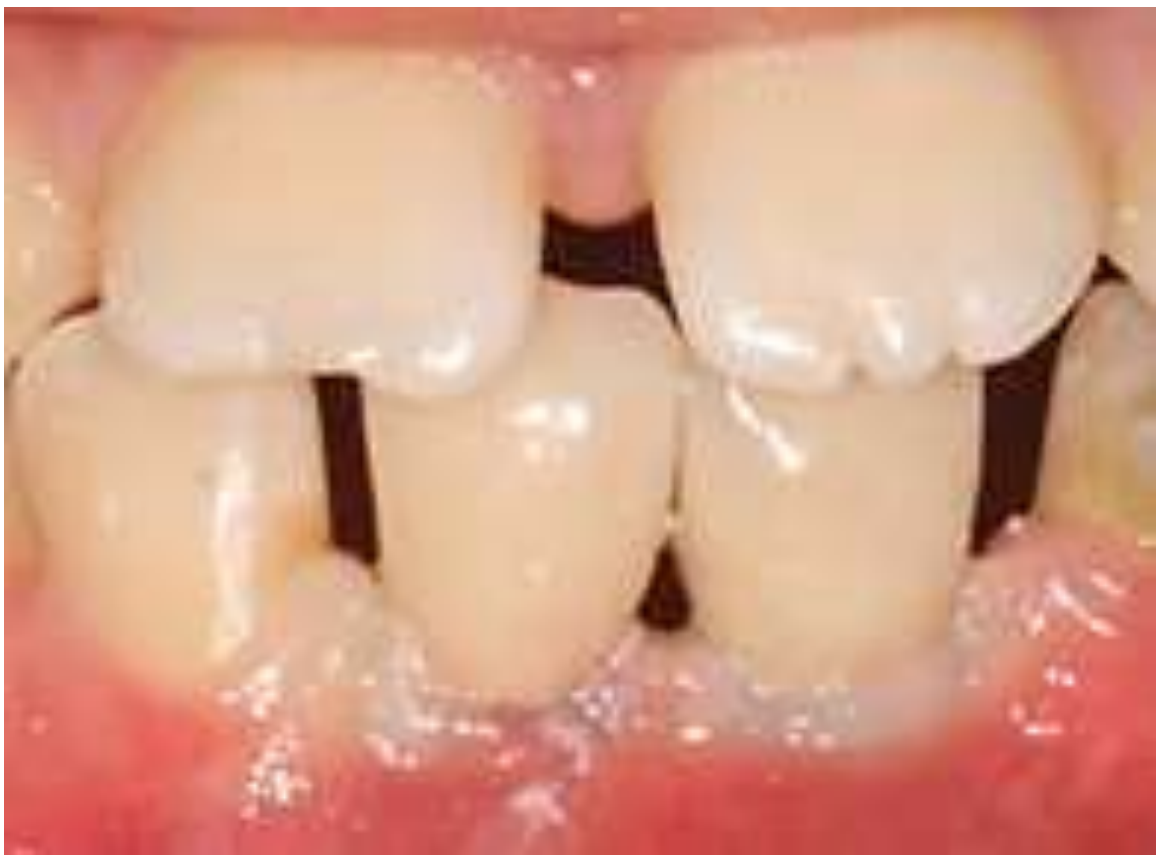

Fig. 2: Fixed prosthetic crowns sealed on the implants. 


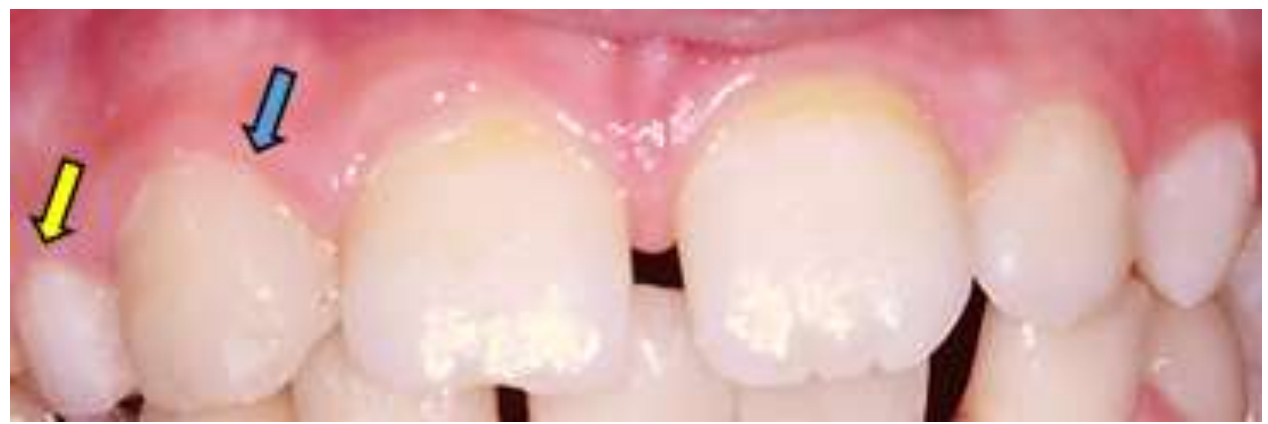

Fig. 3: Coronoplasty of the maxillary permanent right canine (\#13) with composite resin (blue arrow). The maxillary temporary canine (\#53) was conserved on the arch (yellow arrow).

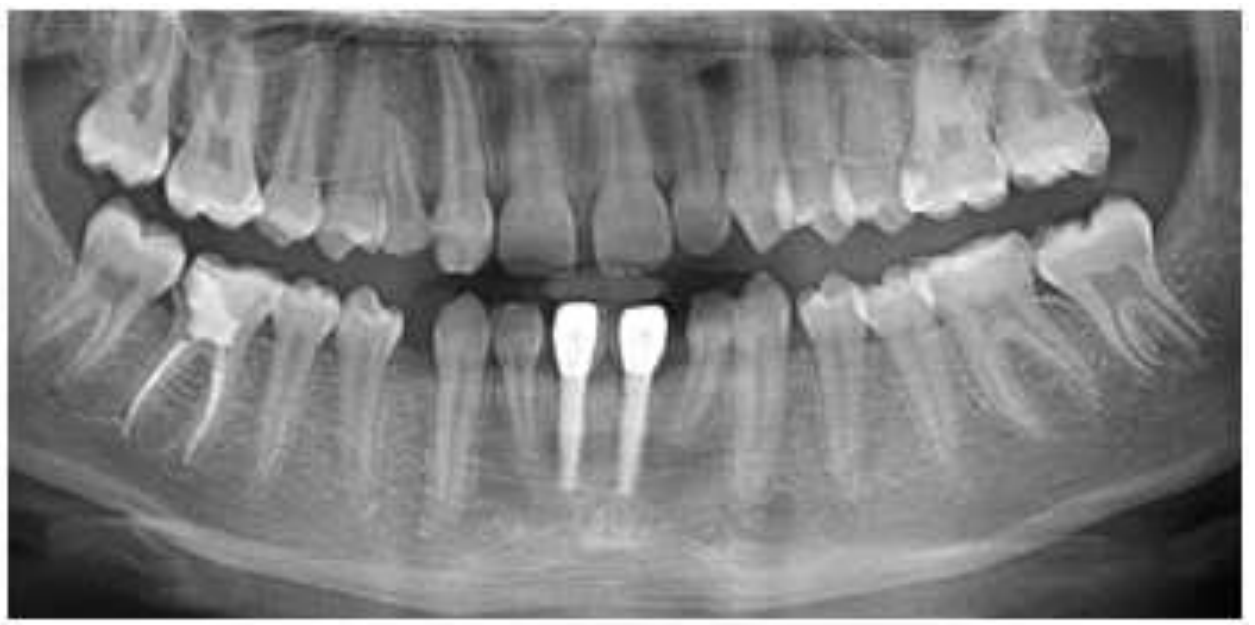

Fig. 4: Orthopantomogram at age fifteen, showing the persistence of the maxillary temporary canine (\#53) in the arch and a stable marginal bone level with no bone loss around the mandibular implants.

\section{Discussion}

Various treatment options for dental agenesis are available (Sfeir et al., 2014). However, the ideal treatment should ensure aesthetic and functional requirements as well as long-term periodontal health (Rosa et al., 2016).

The principal target in this clinical case was reached. Aesthetic and oral function child complains were solved. Since the patient was satisfied and the mini-implants exhibited good long-term results, no modification or updating of the treatment was needed. Even though, orthodontic treatment and placement of implant in the maxilla could be postponed till adult age.

Bone quality and quantity assessment generally defines the characteristics and number of implants. The small diameter of the mini-implants (less than $3 \mathrm{~mm}$ ) allows them to be placed in areas with low bone thickness and makes surgical techniques less invasive and less complex (Marcello-Machado et al., 2018; Bohner et al., 2019). Rapid healing and placement of esthetic prosthetic crowns endorse this type of implant. Placement of the dental implant reduces bone load and delays its resorption (Cronin Jr et al., 2019). In fact, 3 years after implant placement, the marginal bone level was stable with no bone loss.

In the maxilla, to replace the lateral incisor, three treatment options were suggested: Coronoplasty, repositioning of the permanent ectopic canine or placement of an implant. In this case, the position of the permanent canine at the level of the missed lateral incisor, permitted its remodeling without any orthodontic or surgical intervention. Satisfactory result was obtained on the long run with a minimal invasive treatment. Indeed, the repositioning of transposed anterior teeth is very complicated since it can affect the roots and damage the supporting tissues (Tumen et al., 2010).

The conservation of the right temporary maxillary canine on the arch, was essential. In fact, this tooth acted as a space maintainer in the growing patient and allowed preserving the alveolar bone for subsequent implant placement. 
The aesthetic and functional evaluations after a threeyear follow-up were satisfactory. The marginal bone level was stable and the periodontal health, around the mini-implants, were ensured in conjunction with mechanical function (Kinget al., 2016).

\section{Conclusion}

Pediatric dentists face challenge in managing dental agenesis and tooth loss. The use of mini implants is still an important solution reducing the complexity of treatment in growing patient. Aesthetical and functional results improve the child's quality of life and self-esteem.

The treatments must be selected appropriately, respecting the autonomy of the young patient and following the principals of ethics.

\section{Author Contributions}

Roula Berbari: Assessed data and wrote the manuscript.

Nahla Nassif: Helped in writing the paper.

\section{Ethics}

The authors declare no potential conflicts of interest related to authorship and publication of this article.

\section{References}

Al-Ani, A. H., Antoun, J. S., Thomson, W. M., Merriman, T. R., \& Farella, M. (2017). Hypodontia: an update on its etiology, classification and clinical management. BioMed Research International, 2017. https://downloads.hindawi.com/journals/specialissue s/928498.pdf\#page=8

Bohner, L., Hanisch, M., Kleinheinz, J., \& Jung, S. (2019). Dental implants in growing patients: A systematic review. British Journal of Oral and Maxillofacial Surgery, 57(5), 397-406. https://www.sciencedirect.com/science/article/abs/pi i/S0266435619301500

Cronin, R. J., Jr, Oesterle, L. J., \& Ranly, D. M. (1994). Mandibular implants and the growing patient. The International Journal of Oral \& Maxillofacial Implants, 9(1), 55-62. http://www.quintpub.com/userhome/omi/omi_9_1_ Cronin_6.pdf
Fauzi, N. H., Ardini, Y. D., Zainuddin, Z., \& Lestari, W. (2018). A review on non-syndromic tooth agenesis associated with PAX9 mutations. Japanese Dental Science Review, 54(1), 30-36. https://www.sciencedirect.com/science/article/pii/S1 882761616300679

King, P., Maiorana, C., Luthardt, R. G., Sondell, K., Øland, J., Galindo-Moreno, P., \& Nilsson, P. (2016). Clinical and Radiographic Evaluation of a Small-Diameter Dental Implant Used for the Restoration of Patients with Permanent Tooth Agenesis (Hypodontia) in the Maxillary Lateral Incisor and Mandibular Incisor Regions: A 36Month Follow-Up. International Journal of Prosthodontics, 29(2).

Marcello-Machado, R. M., Faot, F., Schuster, A. J., Nascimento, G. G., \& Del Bel Cury, A. A. (2018). Mini-implants and narrow diameter implants as mandibular overdenture retainers: A systematic review and meta-analysis of clinical and radiographic outcomes. Journal of Oral Rehabilitation, 45(2), 161-183.

Nunn, J. H., Carter, N. E., Gillgrass, T. J., Hobson, R. S., Jepson, N. J., Meechan, J. G., \& Nohl, F. S. (2003). The interdisciplinary management of hypodontia: Background and role of paediatric dentistry. British Dental Journal, 194(5), 245-251. https://www.nature.com/articles/4809925

Rosa, M., Lucchi, P., Ferrari, S., Zachrisson, B. U., \& Caprioglio, A. (2016). Congenitally missing maxillary lateral incisors: Long-term periodontal and functional evaluation after orthodontic space closure with first premolar intrusion and canine extrusion. American Journal of Orthodontics and Dentofacial Orthopedics, 149(3), 339-348. https://www.sciencedirect.com/science/article/abs/pi i/S0889540615013037

Sfeir, E., Nassif, N., \& Moukarzel, C. (2014). Use of mini dental implants in ectodermal dysplasia children: follow-up of three cases. Eur J Paediatr Dent, $\quad 15(2 \quad$ Suppl), 207-12. http://admin.ejpd.eu/download/EJPD_2014_2suppl_ 10.pdf

Tumen, D. S., Kaya, F. A., Hamamci, N., Tumen, E. C., \& Berber, G. (2010). Maxillary Canine-Lateral Incisor Transposition: A Case Report. Journal International DentalMedicine Research, 3(2), 75-78. http://www.jidmr.com/journal/DENTISTRY/2010/v ol3_no2/5_D1054_E_Caner_TUMEN.pdf 